\title{
Ruthenium Hydride Catalyzed Direct Oxidation of Alcohols to Carboxylic Acids via Transfer Hydrogenation: Styrene Oxide as Oxygen Source
} \author{
Iraj Mohammadpoor-Baltork \\ Department of Chemistry, Catalysis Division, University of Isfahan, Isfahan 81746-73441, Iran \\ Fax +98(311)6689732; E-mail: moghadamm@sci.ui.ac.ir; E-mail: a.rahmati@sci.ui.ac.ir \\ Received: 07.09.2012; Accepted after revision: 30.10 .2012
}

Behjat Barati, Majid Moghadam,* Abbas Rahmati,* Shahram Tangestaninejad, Valiollah Mirkhani,

\begin{abstract}
Direct oxidation of alcohols to carboxylic acids using styrene epoxide as oxidant in the presence of $\left[\mathrm{RuHCl}(\mathrm{CO})\left(\mathrm{PPh}_{3}\right)_{3}\right]$ complex as catalyst is reported. By this catalytic system, a variety of primary alcohols including substituted benzyl alcohols as well as linear ones were directly converted into carboxylic acids in good to excellent yields.
\end{abstract}

Key words: ruthenium hydride complex, alcohols, carboxylic acids, epoxides, oxidation

Transition-metal hydrides have recently attracted great attention due to their high efficiency and tolerance to functional-group transformation. ${ }^{1}$ In particular, ruthenium hydride catalysts have played a large rule in these important reactions. ${ }^{2}$ However, most of the examples are limited to the reduction of polar functional groups ${ }^{3}$ and reductive carbon-carbon bond formations. ${ }^{2,4}$

Carboxylic acids are key building blocks for the synthesis of a variety of pharmaceutically important compounds ${ }^{5}$ and also have specific industrial applications for production of several compounds. ${ }^{6}$ Transition-metal-catalytic systems have been used for the oxidative dehydrogenation of alcohols to their corresponding carboxylic acids which represents one of the most fundamental transformations in organic chemistry. ${ }^{7}$ Generally, the direct oxidation of alcohols to carboxylic acids is a two-step process; oxidation of the alcohol to the (potentially sensitive) aldehyde and oxidation of the resulting aldehyde to the desired carboxylic acid. Despite recent progress in the direct conversion of primary alcohols into carboxylic acids, the number of methods available is still limited, and often harsh reaction conditions with low functional-group compatibility have to be employed. ${ }^{8}$ But to date, application of transitionmetal hydrides for oxidation of alcohols to carboxylic acids have not been reported. Considering the importance of this reaction and relying pronounced ability of ruthenium hydride complex to influence the reactivity of organic compounds, we decided to explore the ability of $\left[\mathrm{RuHCl}(\mathrm{CO})\left(\mathrm{PPh}_{3}\right)_{3}\right]^{9}$ catalyst in the direct oxidation of alcohols to carboxylic acids in the presence of styrene oxide as oxygen source (Scheme 1).

SYNLETT 2013, 24, 0090-0096

Advanced online publication: 05.12.2012

DOI: 10.1055/s-0032-1317676; Art ID: ST-2012-B0752-L

(c) Georg Thieme Verlag Stuttgart · New York

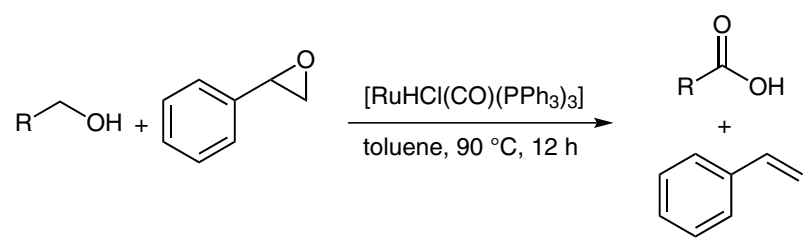

Scheme 1

First, the reaction parameters were optimized in the reaction of benzyl alcohol (1a) with styrene oxide (2a, Table 1). First, the effect of catalyst amount was studied in the model reaction and $5 \mathrm{~mol} \%$ of catalyst was chosen as optimal catalyst amount (Table 1, entry 2). Increasing the amount of catalyst to $10 \mathrm{~mol} \%$ did not affect the yield of product, but decreasing the catalyst amount to $3 \mathrm{~mol} \%$, the yield decreased to $53 \%$ (Table 1, entries 1-3). The effect of solvent was also checked in the model reaction. Among toluene, dichloromethane, dioxane, 1,2-dichloroethane, acetonitrile, and THF as solvents; toluene was chosen as reaction medium because the highest yield was obtained in this solvent (Table 1, entries 2 and 4-8). The ability of other ruthenium catalysts such as $\left[\mathrm{RuH}_{2}(\mathrm{CO})\left(\mathrm{PPh}_{3}\right)_{3}\right]{ }^{10} \quad\left[\mathrm{Ru}\left(\mathrm{O}_{2} \mathrm{CCF}_{3}\right)_{2}(\mathrm{CO})\left(\mathrm{PPh}_{3}\right)_{2}\right],{ }^{11}$ $\left[\mathrm{RuHCl}\left(\mathrm{PPh}_{3}\right)_{3}\right]{ }^{12}\left[\mathrm{RuH}_{2}\left(\mathrm{PPh}_{3}\right)_{3}\right],{ }^{13}$ and $[\mathrm{Ru}(\operatorname{cod})(\cot )]^{14}$ were also investigated, and no better results were obtained in comparison with $\left[\mathrm{RuHCl}(\mathrm{CO})\left(\mathrm{PPh}_{3}\right)_{3}\right]($ Table 1, entries 9-13). It seems that the ease of selective substitution of phosphine ligand trans to $\mathrm{H}^{-}$in cis,mer$\left[\mathrm{RuHCl}(\mathrm{CO})\left(\mathrm{PPh}_{3}\right)_{3}\right]$ by alkoxy ligand (from epoxide) provide clear evidence for the kinetic trans effect of the strong $\sigma$-donor hydrido ligand. ${ }^{15}$ The carbon monoxide, which acts as a weak $\sigma$-donor but a strong $\pi$-acceptor ligand, is ideal for tuning the electronic and steric properties of the Ru center in the reaction intermediates. ${ }^{16}$ The presence of ligands, which increases the electron density on the $\mathrm{Ru}$, reduces the catalytic activity. In the case of $[R u(\operatorname{cod})(\cot )]$ both electronic and steric effects should be considered. ${ }^{17}$ Finally, different epoxides were also applied as oxygen source. The results showed that the best oxygen source is styrene oxide (Table 1, entries 14-19). This can be attributed to increased stability of styrene (as one of the reaction products) relative to the other alkenes which were produced upon oxidation reaction. On the other hand, no progress was observed using $\mathrm{H}_{2} \mathrm{O}_{2}$ or tert- $\mathrm{BuOOH}$ as oxygen source. The scope and generality of this method was checked in the oxidation of a wide range of primary alcohols including benzylic and linear ones using styrene ox- 
Table 1 Optimization of Conditions for the Reaction of $\mathbf{1 a}$ with $\mathbf{2}^{\mathrm{a}}$

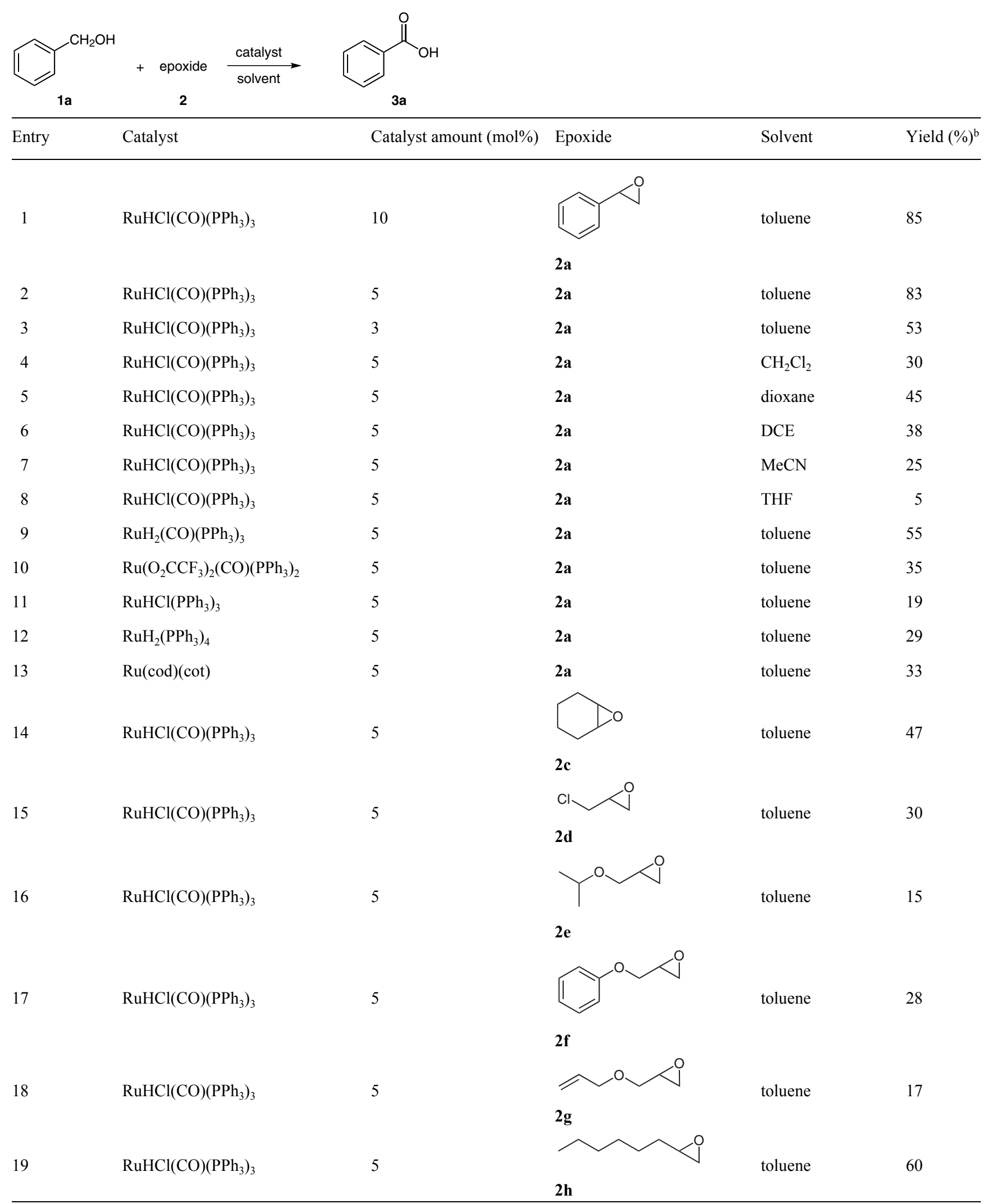

${ }^{\text {a }}$ All reactions were performed using $\mathbf{1 a}(1 \mathrm{mmol}), \mathbf{2}(1.3 \mathrm{mmol})$, catalyst, and solvent $(2 \mathrm{~mL})$ for $12 \mathrm{~h}$ in a screw capped test tube at $90{ }^{\circ} \mathrm{C}$.

${ }^{\mathrm{b}}$ The yields refer to pure isolated products. 


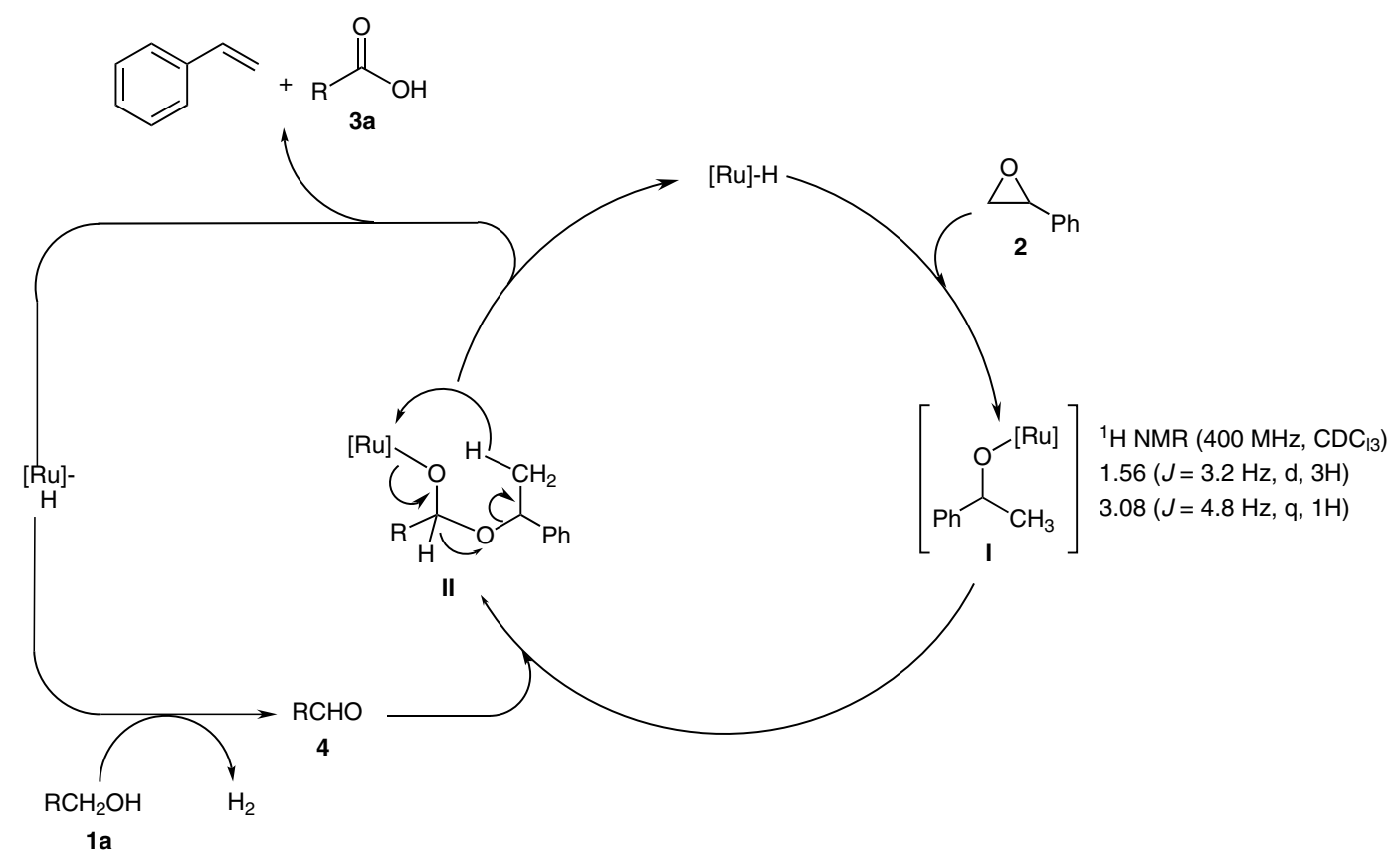

Scheme 2

ide as oxygen source (Table 2). Different benzyl alcohols bearing electron-withdrawing and electron-donating substituents were efficiently converted into their corresponding substituted benzoic acids (Table 2 , entries $1-5,12$, and 13). It seems that the nature of the substituents has no significant effect on the product yield. Surprisingly, upon oxidation of 4-methoxybenzyl alcohol (11), the 4hydroxybenzoic acid (3I) was obtained as product, and the methyl ether was deprotected to its corresponding hydroxy compound (Table 2, entry 13).

A series of substituted $N$-(2-hydroxyethyl)benzamides were also oxidized to their corresponding carboxylic acids in high to excellent yields with styrene oxide in the presence of $\left[\mathrm{RuHCl}(\mathrm{CO})\left(\mathrm{PPh}_{3}\right)_{3}\right]$ (Table 2, entries 7-10). Cinnamyl alcohol as a linear alcohol was also oxidized successfully to cinnamic acid in high yield (Table 2, entry 11).

Despite the ability of this catalyst in the catalyzing the Tishchenko reaction, ${ }^{21}$ no Tishchenko products were observed in the presence of styrene oxide (even for compound 1f).

The plausible mechanism for this reaction is as following: First, the $\left[\mathrm{RuHCl}(\mathrm{CO})\left(\mathrm{PPh}_{3}\right)_{3}\right]$ reacts with styrene oxide $\mathbf{2}$, and a hydroruthenation reaction gives the ruthenium complex I. The structure of complex I was confirmed by ${ }^{1} \mathrm{H}$ NMR and ${ }^{13} \mathrm{C}$ NMR spectroscopy. As mentioned previously, the alcohol 1 can be converted into aldehyde 4 by $\left[\mathrm{RuHCl}(\mathrm{CO})\left(\mathrm{PPh}_{3}\right)_{3}\right]$ and releases $\mathrm{H}_{2}$ gas..$^{2 \mathrm{k}}$ The resulting complex I would undergo a nucleophilic reaction with aldehyde $\mathbf{4}$ to give the ruthenium complex II, which in turns converts into styrene and carboxylic acid 3, the $[\mathrm{RuH}-$
$\left.\mathrm{Cl}(\mathrm{CO})\left(\mathrm{PPh}_{3}\right)_{3}\right]$ releases, and the catalytic cycle will continue (Scheme 2).

Further investigations showed that, in addition to styrene, ethylbenzene is also present in the reaction mixture. This can be attributed to the fact that the produced styrene acts as a hydrogen accepter (Scheme 3$).{ }^{18}$

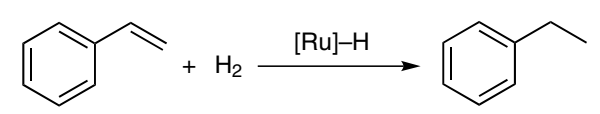

Scheme 3

On the other hand, excess styrene oxide was not detected in the reaction mixture, but a small amount of 1-phenylethanol was. It seems that the styrene oxide can also act as hydrogen acceptor (Scheme 4).<smiles>CC(O)c1ccccc1</smiles>

Scheme 4

To stress this point, the oxidation of benzaldehyde with styrene oxide was also investigated in the presence of $\left[\mathrm{RuHCl}(\mathrm{CO})\left(\mathrm{PPh}_{3}\right)_{3}\right]$. The results showed that the benzoic acid was obtained in $80 \%$ after eight hours, and styrene was produced as byproduct. These observations show that benzyl alcohol should be converted firstly into benzaldehyde and then converted into benzoic acid. All these observations showed that the proposed mechanism is reasonable. 
Table 2 Ruthenium Hydride Catalyzed Direct Oxidation of Alcohols to Carboxylic Acids with Styrene Oxide ${ }^{\mathrm{a}}$

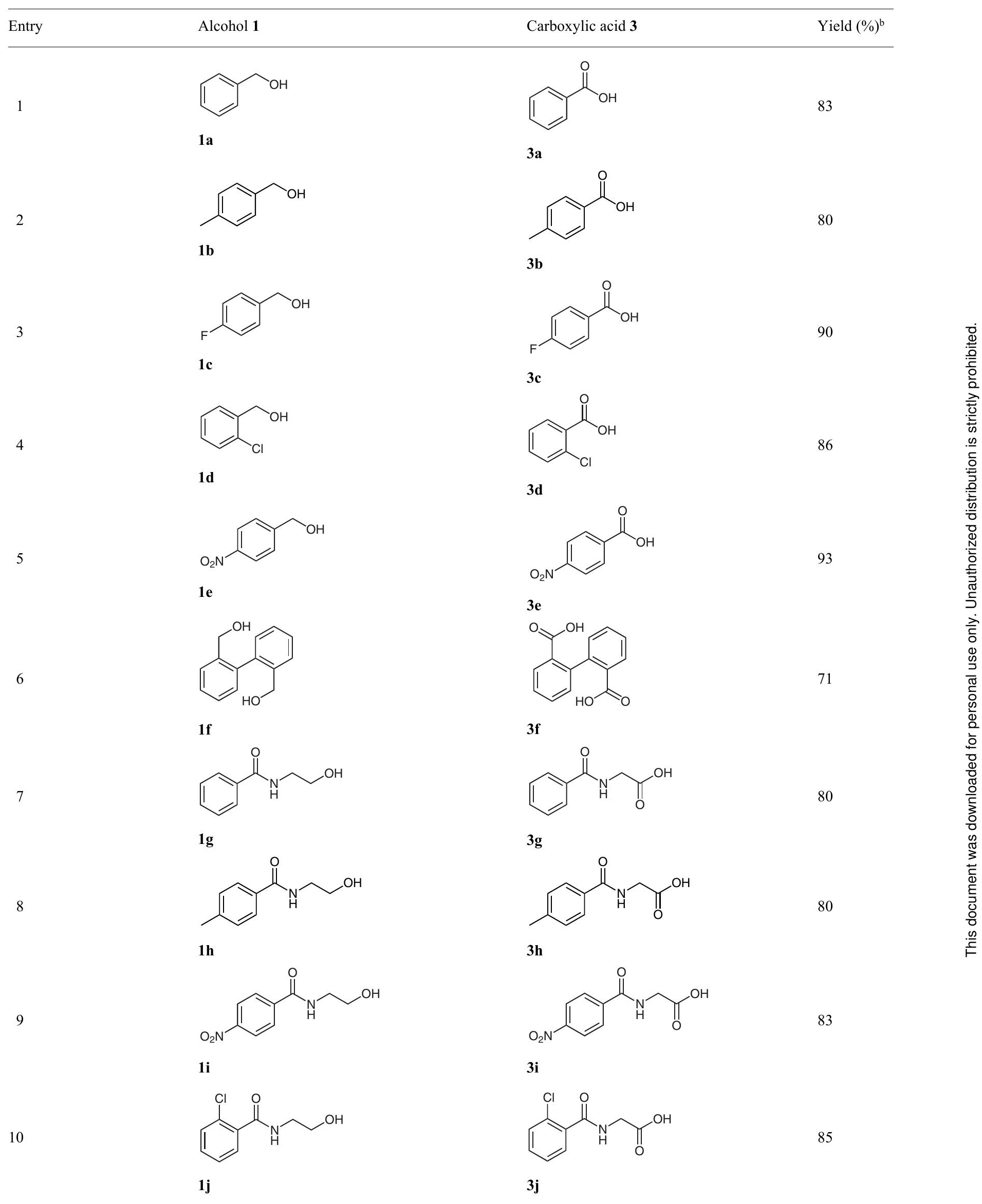


Table 2 Ruthenium Hydride Catalyzed Direct Oxidation of Alcohols to Carboxylic Acids with Styrene Oxide ${ }^{\text {(continued) }}$

Entry

${ }^{a}$ All reactions were performed using alcohol $(1 \mathrm{mmol})$, styrene epoxide $(1.3 \mathrm{mmol})$, and catalyst $(5 \mathrm{~mol} \%)$ in toluene $(2 \mathrm{~mL})$ at $90{ }^{\circ} \mathrm{C}$ for $12 \mathrm{~h}$.

${ }^{\mathrm{b}}$ The yields refer to pure isolated products.

In conclusion, in the present work an efficient and new method for direct oxidation of alcohols to carboxylic acids is reported. In the presence of $\left[\mathrm{RuHCl}(\mathrm{CO})\left(\mathrm{PPh}_{3}\right)_{3}\right] \mathrm{com}-$ plex as catalyst different primary alcohols including substituted benzyl alcohols and also linear ones were directly converted into carboxylic acids in good to excellent yields.

Typical Procedure for the Ruthenium Hydride Catalyzed Oxidation of Benzyl Alcohol to Benzoic Acid

Benzyl alcohol (1a, $108.1 \mathrm{mg}, 1.0 \mathrm{mmol})$, styrene epoxide (2, 156.2 $\mathrm{mg}, 1.3 \mathrm{mmol})$, [RuHCl$\left.(\mathrm{CO})\left(\mathrm{PPh}_{3}\right)_{3}\right](50.9 \mathrm{mg}, 0.052 \mathrm{mmol})$, and toluene $(2 \mathrm{~mL})$ were mixed in a screw-capped test tube and purged with argon and sealed. The mixture was stirred at $90{ }^{\circ} \mathrm{C}$ for $12 \mathrm{~h}$. After the reaction was completed, the solvent was removed under reduce pressure. The residue was purified by chromatography on silica gel plate (PE-EtOAc, 6:1), and the pure compound 3a was obtained as a white crystal after recrystallization from $\mathrm{H}_{2} \mathrm{O}-\mathrm{MeOH}$ (101.4 mg, 83\%).

\section{Spectral Data}

\section{1-Benzoic Acid (3a)}

Colorless crystals; yield $101.4 \mathrm{mg}, 83 \%$, mp $121-123{ }^{\circ} \mathrm{C} .{ }^{1} \mathrm{H}$ NMR $\left(400 \mathrm{MHz}, \mathrm{DMSO}-d_{6}\right): \delta=7.51(\mathrm{t}, 2 \mathrm{H}, J=16 \mathrm{~Hz}), 7.63(\mathrm{t}, 1 \mathrm{H}, J$ $=8 \mathrm{~Hz}), 7.95(\mathrm{~d}, 2 \mathrm{H}, J=4 \mathrm{~Hz}), 12.99(\mathrm{br} \mathrm{s}, 1 \mathrm{H}) .{ }^{13} \mathrm{C}$ NMR $(100$ $\left.\mathrm{MHz}, \mathrm{DMSO}-d_{6}\right): \delta=128.55,129.23,130.69,132.58,167.30$.

\section{4-Methylbenzoic Acid (3b)}

White solid; yield $109.5 \mathrm{mg}, 80 \%$; mp $178-179{ }^{\circ} \mathrm{C} .{ }^{1} \mathrm{H}$ NMR (400 $\left.\mathrm{MHz}, \mathrm{DMSO}-d_{6}\right): \delta=2.37(\mathrm{~s}, 1 \mathrm{H}), 7.31(\mathrm{~d}, 2 \mathrm{H}, J=8 \mathrm{~Hz}), 7.84(\mathrm{~d}$, $2 \mathrm{H}, J=8 \mathrm{~Hz}), 12.82$ (br s, $1 \mathrm{H}) .{ }^{13} \mathrm{C}$ NMR $\left(100 \mathrm{MHz}, \mathrm{DMSO}-d_{6}\right)$ : $\delta=21.10,127.96,129.10,129.30,143.01,167.29$.

\section{4-Fluorobenzoic Acid (3c)}

White solid; yield $126.1,90 \%$; mp $182-184{ }^{\circ} \mathrm{C} .{ }^{1} \mathrm{H}$ NMR $(400$ MHz, DMSO- $\left.d_{6}\right): \delta=7.33$ (t, $\left.2 \mathrm{H}, J=8 \mathrm{~Hz}\right), 7.99-8.03(\mathrm{~m}, 2 \mathrm{H})$, 13.07 (br s, $1 \mathrm{H}) .{ }^{13} \mathrm{C}$ NMR (100 MHz, DMSO- $\left.d_{6}\right): \delta=115.72$, 127.26, 132.13, 163.61, 166.11.

\section{2-Chlorobenzoic Acid (3d)}

White solid; yield $134.6 \mathrm{mg}, 86 \%$; mp $137-138^{\circ} \mathrm{C} .{ }^{1} \mathrm{H}$ NMR $(400$ MHz, DMSO- $\left.d_{6}\right): \delta=7.42-7.55(\mathrm{~m}, 3 \mathrm{H}), 7.79(\mathrm{~d}, 2 \mathrm{H}, J=8 \mathrm{~Hz})$, 13.44 (br s, $1 \mathrm{H}) \cdot{ }^{13} \mathrm{C}$ NMR (100 MHz, DMSO- $\left.d_{6}\right): \delta=127.21$, $130.58,130.76,131.50,132.56,166.28$.

\section{4-Nitrobenzoic Acid (3e)}

White solid; yield $155.4 \mathrm{mg}, 93 \%$; mp $237-239^{\circ} \mathrm{C} .{ }^{1} \mathrm{H}$ NMR (400 MHz, DMSO- $\left.d_{6}\right): \delta=8.27(\mathrm{~d}, 2 \mathrm{H}, J=8 \mathrm{~Hz}), 8.38(\mathrm{~d}, 2 \mathrm{H}, J=8$ $\mathrm{Hz}), 12.92$ (br s, $1 \mathrm{H}) .{ }^{13} \mathrm{C}$ NMR (100 MHz, DMSO- $\left.d_{6}\right): \delta=183.4$, 151.6, 133.9, 130.4, 124.7.

\section{2,2'-Biphenyldicarboxylic Acid (3f)}

White solid; yield $172.0 \mathrm{mg}, 71 \%$; mp 224-226 ${ }^{\circ} \mathrm{C}$. IR (KBr): 3064 , 2994, 2885, 2818, 2645, 2567, 1686, 1597, 1577, 1453, 1407, 1297, $1273,1136,1049,1105,1049,1003,921,796,754,703 \mathrm{~cm}^{-1} .{ }^{1} \mathrm{H}$ NMR (400 MHz, DMSO- $\left.d_{6}\right): \delta=6.94-8.16(\mathrm{~m}, 8 \mathrm{H}), 12.47$ (br s, 2 H). ${ }^{13} \mathrm{C} \mathrm{NMR}\left(100 \mathrm{MHz}, \mathrm{DMSO}-d_{6}\right): \delta=126.9,129.4,130.3,131.0$, 142.9, 167.9. MS (EI): $m / z(\%)=242(19)\left[\mathrm{M}^{+}\right], 197(100), 181$ (28), 152 (46), 139 (13), 115 (18), 98 (6), 89 (3), 76 (11). Anal. Calcd for $\mathrm{C}_{14} \mathrm{H}_{10} \mathrm{O}_{4}: \mathrm{C}, 69.42 ; \mathrm{H}, 4.16 ; \mathrm{N}, 5.78$. Found: $\mathrm{C}, 69.23 ; \mathrm{H}$, $4.06 ; \mathrm{N}, 5.68$.

\section{2-Benzamidoacetic Acid (3g)}

White solid; yield $143.3 \mathrm{mg}, 80 \%$; $\mathrm{mp} 185-188^{\circ} \mathrm{C}$. IR (KBr): 3343 , $3075,2938,1742,1602,1556,1490,1416,1395,1336,1317,1305$, $1257,1181,1078,999,943,849,806,723 \mathrm{~cm}^{-1} .{ }^{1} \mathrm{H}$ NMR $(400$ MHz, DMSO- $\left.d_{6}\right): \delta=3.95(\mathrm{~d}, 2 \mathrm{H}, J=4 \mathrm{~Hz}), 7.49(\mathrm{t}, 2 \mathrm{H}, J=8 \mathrm{~Hz})$, $7.55(\mathrm{t}, 2 \mathrm{H}, J=4 \mathrm{~Hz}), 7.89(\mathrm{~d}, 1 \mathrm{H}, J=8 \mathrm{~Hz}), 8.87(\mathrm{t}, 1 \mathrm{H}, J=6$ $\mathrm{Hz}), 12.64$ (br s, $1 \mathrm{H}) \cdot{ }^{13} \mathrm{C}$ NMR (100 MHz, DMSO- $\left.d_{6}\right): \delta=41.2$, 127.2, 128.2, 128.3, 131.4, 133.7, 166.4, 171.3. MS (EI): $\mathrm{m} / z(\%)=$ $179(10)\left[\mathrm{M}^{+}\right], 135$ (82), 104 (100), 83 (67), 77 (95), 69 (74), 57 (82), 51 (87), 45 (82), 43 (81), 41 (74). Anal. Calcd for $\mathrm{C}_{9} \mathrm{H}_{9} \mathrm{NO}_{3}$ : C, 60.34; H, 5.06; N, 7.82. Found: C, 60.22; H, 4.96; N, 7.69.

\section{2-(4-Methylbenzamido)acetic Acid (3h)}

White solid; yield $154.5 \mathrm{mg}, 80 \%$; mp $153-155^{\circ} \mathrm{C}$. IR (KBr): 3354 , 2986, 1746, 1680, 1613, 1556, 1506, 1416, 1325, 1260, 1214, 1121, $1000,833,755 \mathrm{~cm}^{-1} .{ }^{1} \mathrm{H}$ NMR (400 MHz, DMSO- $\left.d_{6}\right): \delta=2.36(\mathrm{~s}$, $3 \mathrm{H}), 3.91(\mathrm{~d}, 2 \mathrm{H}, J=8 \mathrm{~Hz}), 7.29(\mathrm{~d}, 2 \mathrm{H}, J=8 \mathrm{~Hz}), 7.78(\mathrm{~d}, 2 \mathrm{H}$, $J=8 \mathrm{~Hz}), 8.77(\mathrm{t}, 1 \mathrm{H}, J=4 \mathrm{~Hz}), 12.63(\mathrm{~s}, 1 \mathrm{H}) .{ }^{13} \mathrm{C}$ NMR $(100$ MHz, DMSO- $\left.d_{6}\right): \delta=20.93,41.33,127.2,128.8,131.0,141.3$, 166.3, 171.4. MS (EI): $m / z(\%)=193(1)\left[\mathrm{M}^{+}\right], 149(37), 119(100)$, 
91 (80), 65 (38). Anal. Calcd for $\mathrm{C}_{10} \mathrm{H}_{11} \mathrm{NO}_{3}: \mathrm{C}, 62.17 ; \mathrm{H}, 5.74 ; \mathrm{N}$, 7.25. Found: C, 61.98; H, 5.63; N, 7.05.

\section{2-(4-Nitrobenzamido)acetic Acid (3i)}

Light brown solid; yield $186.1 \mathrm{mg}, 83 \%$; mp $152-154{ }^{\circ} \mathrm{C}$. IR $(\mathrm{KBr})$ : 3316, 3110, 1706, 1641, 1601, 1541, 1425, 1351, 1297, 1231, 1108, 1013, 930, 874, 831, 801, 786, $716 \mathrm{~cm}^{-1} .{ }^{1} \mathrm{H}$ NMR $(400 \mathrm{MHz}$ DMSO- $\left.d_{6}\right): \delta=3.97(\mathrm{~d}, 2 \mathrm{H}, J=8 \mathrm{~Hz}), 8.11(\mathrm{~d}, 2 \mathrm{H}, J=8 \mathrm{~Hz}), 8.33$ $(\mathrm{d}, 2 \mathrm{H}, J=8 \mathrm{~Hz}), 9.28(\mathrm{t}, 1 \mathrm{H}, J=5 \mathrm{~Hz}), 13.14(\mathrm{br} \mathrm{s}, 1 \mathrm{H}) .{ }^{13} \mathrm{C} \mathrm{NMR}$ $\left(100 \mathrm{MHz}, \mathrm{DMSO}-d_{6}\right): \delta=41.3,123.6,128.8,139.3,149.1,164.8$, 170.9. MS (EI): $m / z(\%)=224(2)\left[\mathrm{M}^{+}\right],(179,29), 150(100), 120$ (24), 104 (91), 92 (62), 50 (83). Anal. Calcd for $\mathrm{C}_{9} \mathrm{H}_{8} \mathrm{~N}_{2} \mathrm{O}_{5}$ : C, 48.22; H, 3.60; N, 12.50. Found: C, 48.12; H, 3.47; N, 12.27.

\section{2-(2-Chlorobenzamido)acetic Acid (3j)}

White solid; yield $181.6 \mathrm{mg}, 85 \%$; mp $158-162{ }^{\circ} \mathrm{C}$. IR (KBr): 3289 , $3082,1721,1626,1597,1596,1553,1469,1437,1402,1350,1321$, $1259,1230,1173,1052,999,947,842,760 \mathrm{~cm}^{-1} .{ }^{1} \mathrm{H}$ NMR $(400$ MHz, DMSO- $\left.d_{6}\right): \delta=3.92(\mathrm{~d}, 2 \mathrm{H}, J=8 \mathrm{~Hz}), 7.40-7.49(\mathrm{~m}, 3 \mathrm{H})$, $7.51(\mathrm{t}, 1 \mathrm{H}, J=4 \mathrm{~Hz}), 8.79(\mathrm{t}, 1 \mathrm{H}, J=4 \mathrm{~Hz}), 12.71(\mathrm{br} \mathrm{s}, 1 \mathrm{H}) .{ }^{13} \mathrm{C}$ NMR $\left(100 \mathrm{MHz}, \mathrm{DMSO}-d_{6}\right): \delta=40.9,127.0,129.0,129.9,130.8$, 132.6, 136.2, 166.6, 170.9. MS (EI): $m / z(\%)=213(1)\left[\mathrm{M}^{+}\right], 168$ (34), 139 (100), 111 (47), 75 (38), 50 (17). Anal. Calcd for $\mathrm{C}_{9} \mathrm{H}_{8} \mathrm{NO}_{3} \mathrm{Cl}: \mathrm{C}, 50.60 ; \mathrm{H}, 3.77 ; \mathrm{N}, 6.56$. Found: $\mathrm{C}, 50.45 ; \mathrm{H}, 3.60$; N, 6.46 .

\section{Cinnamic Acid (3k)}

White solid; yield $117.5 \mathrm{mg}, 79 \%$; mp $129-132{ }^{\circ} \mathrm{C}$. ${ }^{1} \mathrm{H}$ NMR $(400$ MHz, DMSO- $\left.d_{6}\right): \delta=6.56(\mathrm{~d}, 1 \mathrm{H}, J=8 \mathrm{~Hz}), 7.43-7.73(\mathrm{~m}, 5 \mathrm{H})$, $7.90(\mathrm{~d}, 1 \mathrm{H}, J=8 \mathrm{~Hz}), 11.61(\mathrm{br} \mathrm{s}, 1 \mathrm{H}) .{ }^{13} \mathrm{C} \mathrm{NMR}(100 \mathrm{MHz}$, DMSO- $\left.d_{6}\right): \delta=173.9,147.16,135.1,130.9,128.9,128.3,118.4$.

\section{4-Hydroxybenzoic Acid (3I)}

White solid; yield $114.6 \mathrm{mg}, 83 \%$; mp $210-214{ }^{\circ} \mathrm{C}$. ${ }^{1} \mathrm{H}$ NMR $(400$ MHz, DMSO- $\left.d_{6}\right): \delta=6.93(\mathrm{~d}, 2 \mathrm{H}, J=8.4 \mathrm{~Hz}), 7.77(\mathrm{~d}, 2 \mathrm{H}, J=8.4$ $\mathrm{Hz}), 9.79$ (s, $1 \mathrm{H}), 10.63$ (br s, $1 \mathrm{H}) .{ }^{13} \mathrm{C}$ NMR (100 MHz, DMSO$\left.d_{6}\right): \delta=115.8,128.4,132.1,163.3,191.0$.

\section{Formation of Ruthenium Complex I:}

\section{$\left\{\mathrm{Ru}\left[\mathrm{OCH}\left(\mathrm{CH}_{3}\right) \mathrm{Ph}\right] \mathrm{Cl}(\mathrm{CO})\left(\mathrm{PPh}_{3}\right)_{2}\right\}$}

$\mathrm{RuHCl}(\mathrm{CO})\left(\mathrm{PPh}_{3}\right)_{3}(0.05 \mathrm{mmol})$ and $\mathrm{CDCl}_{3}(1 \mathrm{~mL})$ were placed in an NMR tube. The tube was purged with $\mathrm{N}_{2}$, capped with a rubber septum, and heated at $90{ }^{\circ} \mathrm{C}$ for $10 \mathrm{~min}$. After cooling to r.t., styrene oxide $(\mathbf{2 a}, 0.05 \mathrm{mmol})$ was added. The resulting mixture was heated at $90{ }^{\circ} \mathrm{C}$ for $30 \mathrm{~min}$. The formation of complex I was confirmed by ${ }^{1} \mathrm{H}$ NMR and ${ }^{13} \mathrm{C}$ NMR measurements. Recrystallization from $\mathrm{CHCl}_{3}$ and hexane gave the ruthenium complex $\mathbf{I}$.

White solid. IR (KBr): $v_{\mathrm{CO}}=1920 \mathrm{~cm}^{-1}$. ${ }^{1} \mathrm{H}$ NMR $(400 \mathrm{MHz}$, $\left.\mathrm{CDCl}_{3}\right): \delta=1.56(\mathrm{~d}, 3 \mathrm{H}, J=3.2 \mathrm{~Hz}), 3.08(\mathrm{q}, 1 \mathrm{H}, J=4.8 \mathrm{~Hz}), 7.38$ $7.42(\mathrm{~m}, 17 \mathrm{H}), 7.51-7.55(\mathrm{~m}, 6 \mathrm{H}), 7.59-7.64(\mathrm{~m}, 6 \mathrm{H}), 7.73-7.76$ $(\mathrm{m}, 6 \mathrm{H}) .{ }^{13} \mathrm{C}$ NMR $\left(125 \mathrm{MHz}, \mathrm{CDCl}_{3}\right): \delta=18.27,51.09,125.34$, $127.71,128.10,129.97\left[\mathrm{~d},{ }^{3} J(\mathrm{C}-\mathrm{P})=7 \mathrm{~Hz}\right], 130.42\left[\mathrm{~d},{ }^{4} J(\mathrm{C}-\mathrm{P})=2\right.$ $\mathrm{Hz}], 131.84\left[\mathrm{~d},{ }^{2} J(\mathrm{C}-\mathrm{P})=14 \mathrm{~Hz}\right], 134.42\left[\mathrm{~d},{ }^{1} J(\mathrm{C}-\mathrm{P})=43 \mathrm{~Hz}\right]$, 207.17 (t, $J=9.5 \mathrm{~Hz}) .{ }^{31} \mathrm{P} \mathrm{NMR}\left(160 \mathrm{MHz}, \mathrm{CDCl}_{3}\right): \delta=35.95$, 38.96. Anal. Calcd for $\mathrm{C}_{45} \mathrm{H}_{39} \mathrm{ClO}_{2} \mathrm{P}_{2} \mathrm{Ru}: \mathrm{C}, 66.70 ; \mathrm{H}, 4.85$. Found: C, 66.47; H, 4.83.

\section{Acknowledgment}

The financial support of this work by the Research Council of The University of Isfahan is acknowledged.

Supporting Information for this article is available online at http://www.thieme-connect.com/ejournals/toc/synlett.

\section{References}

(1) (a) Transition Metals for Organic Synthesis: Building Blocks and Fine Chemicals; Vol. 1 and 2; Beller, M.; Bolm, C., Eds.; Wiley-VCH: Weinheim, 1998. (b) Davies, S. G. Organotransition Metal Chemistry: Application to Organic Synthesis; Pergamon Press: Oxford, 1982, Chap. 6.

(2) (a) Michalak, M.; Wicha, J. Synlett 2005, 2277. (b) Doi, T.; Fukuyama, T.; Minamino, S.; Husson, G.; Ryu, I. Chem. Commun. 2006, 1875. (c) Krompiec, S.; Kuznik, N.; Urbala, M.; He, R. J. Mol. Catal. A: Chem. 2006, 256, 17. (d) Doi, T.; Fukuyama, T.; Horiguchi, J.; Okamura, T.; Ryu, I. Synlett 2006, 721. (e) Doi, T.; Fukuyama, T.; Minamino, S.; Ryu, I. Synlett 2006, 3013. (f) Arisawa, M.; Terada, Y.; Takahashi, K.; Nakagawa, M.; Nishida, A. J. Org. Chem. 2006, 71, 4255. (g) Burling, S.; Paine, B. M.; Nama, D.; Brown, V. S.; Mahon, M. F.; Prior, T. J.; Pregosin, P. S.; Whittlesey, M. K.; Williams, J. M. J. J. Am. Chem. Soc. 2007, 129, 1987. (h) Casey, C. P.; Clark, T. B.; Guzei, A. J. Am. Chem. Soc. 2007, 129, 11821. (i) Fukuyama, T.; Doi, T.; Minamino, S.; Omura, S.; Ryu, I. Angew. Chem. Int. Ed. 2007, 46, 5559. (j) Omura, S.; Fukuyama, T.; Horiguchi, J.; Murakami, Y.; Ryu, I. J. Am. Chem. Soc. 2008, 130, 14094. (k) Shibahara, F.; Bower, J. F.; Krische, M. J. J. Am. Chem. Soc. 2008, 130, 6338. (1) Omura, S.; Fukuyama, T.; Murakami, Y.; Okamoto, H.; Ryu, I. Chem. Commun. 2009, 6741 .

(3) (a) Denichoux, A. I.; Fukuyama, T.; Doi, T.; Horiguchi, J.; Ryu, I. Org. Lett. 2010, 12, 1. (b) Patman, R. L.; Williams, V. M.; Bower, J. F.; Krische, M. J. Angew. Chem. Int. Ed. 2008, 47,5220 .

(4) (a) Wakamatsu, H.; Nishida, M.; Adachi, N.; Mori, M. J. Org. Chem. 2000, 65, 3966. (b) Krompiec, S.; Pigulla, M.; Szczepankiewicz, W.; Bieg, T.; Kuznik, N.; LeszczynskaSejda, K.; Kubicki, M.; Borowiak, T. Tetrahedron Lett. 2001, 42, 7095. (c) Kuznik, N.; Krompiec, S.; Bieg, T.; Baj, S.; Skutil, K.; Chrobok, K. J. Organomet. Chem. 2003, 665, 167. (d) Shibahara, F.; Bower, J. F.; Krische, M. J. J. Am. Chem. Soc. 2008, 130, 14120. (e) Ngai, M.-Y.; Skucas, E.; Krische, M. J. Org. Lett. 2008, 10, 2705. (f) Smejkat, T.; Han, H.; Breit, B.; Krische, J. J. Am. Chem. Soc. 2009, 131, 10366.

(5) (a) Kornievskaja, V. S.; Kruppa, A. I.; Leshina, T. V. J. Inclusion Phenom. Macrocyclic Chem. 2008, 60, 123. (b) Marinello, J.; Marchand, C.; Mott, B. T.; Bain, A.; Thomas, C. J.; Pommier, Y. Biochemistry 2008, 47, 9345. (c) Polyakov, N. E.; Leshina, T. V. Open Conf. Proc. J. 2011, 2, 64. (d) Vippagunta, S. R.; Brittain, H. G.; Grant, D. J. W. Adv. Drug Delivery Rev. 2001, 48, 3. (e) Pierre, F.; Chua, P. C.; O’Brien, S. E.; Siddiqui-Jain, A.; Bourbon, P.; Haddach, M.; Michaux, J.; Nagasawa, J.; Schwaebe, M. K.; Stefan, E.; Vialettes, A.; Whitten, J. P.; Chen, T. K.; Darjania, L.; Stansfield, R.; Anderes, K.; Bliesath, J.; Drygin, D.; Ho, C.; Omori, M.; Proffitt, C.; Streiner, N.; Trent, K.; Rice, W. G.; Ryckman, D. M. J. Med. Chem. 2011, 54, 635.

(6) (a) King, C. J. Chemtech. 1992, 285. (b) Kirk, R. E.; Othmer, D. F. Encyclopedia of Chemical Technology, 4th ed.; Wiley: New York, 1995. (c) Hong, Y. K.; Hong, W. H.; Han, D. H. Biotechnol. Bioprocess Eng. 2001, 6, 386. (d) Yu, L.; Lin, T.; Guo, Q.; Hao, J. Desalination 2003, 154. (e) Saito, S.; Nakasato, K.; Katoh, Y.; Oshibe, Y.; Ishidoya, M. Mater. Trans. 2004, 45, 759. (f) Wang, Z.; Luo, Y.; Yu, P. J. Memb. Sci. 2006, 280. (g) Ferrer, J. S. J.; Laborie, S.; Durand, G.; Rakib, M. J. Memb. Sci. 2006, 280. (h) Vertova, A.; Aricci, G.; Rondinini, S.; Miglio, R.; Carnelli, L.; D’Olimpio, P. J. Appl. Electrochem. 2009, 39, 2051. 
(i) Reddy, N.; Li, Y.; Yang, Y. Biotechnol. Prog. 2009, 25, 139.

(7) (a) Mannam, S.; Sekar, G. Tetrahedron Lett. 2008, 49, 2457. (b) Yasuda, K.; Ley, S. V. J. Chem. Soc., Perkin Trans. 1 2002, 1024. (c) Donze, C.; Korovchenko, P.; Gallezot, P.; Besson, M. Appl. Catal., B 2007, 70, 621. (d) Schmidt, A.-K. C.; Stark, C. B. W. Org. Lett. 2011, 13, 4164.

(8) For selected methodological approaches, see: (a) Hunsen, M. Synthesis 2005, 2487. (b) Tashino, Y.; Togo, H. Synlett 2004, 2010. (c) Travis, B. R.; Sivakumar, M.; Hollist, G. O.; Borhan, B. Org. Lett. 2003, 5, 1031. (d) Yasuda, K.; Ley, S. V. J. Chem. Soc., Perkin Trans. 1 2002, 1024.

(e) Mazitschek, R.; Mülbaier, M.; Giannis, A. Angew. Chem. Int. Ed. 2002, 41, 4059; Angew. Chem. 2002, 114, 4216.

(9) (a) Ahmad, N.; Levison, J. J.; Robinson, S. D.; Uttley, M. F. Inorg. Synth. 1974, 15, 45. (b) Levison, J. J.; Robinson, S. D. J. Chem. Soc. A 1970, 2947.
(10) (a) Robinson, S. D. Inorg. Chem. 1977, 16, 137. (b) Ahmad, N.; Levison, J. J.; Robinson, S. D.; Utteley, M. F. Inorg. Synth. 1974, 15, 48.

(11) (a) Robinson, S. D.; Uttley, M. F. J. Chem. Soc., Dalton Trans. 1973, 1912. (b) Dobson, A.; Robinson, S. D. J. Organomet. Chem. 1975, 87, C52. (c) Dobson, A.; Robinson, S. D.; Uttley, M. F. J. Chem. Soc., Dalton Trans. 1975, 370. (d) Dobson, A.; Robinson, S. D. Inorg. Chem. 1977, 16, 1321.

(12) Bianchi, M.; Matteoli, U.; Frediani, P.; Menchi, G.; Otteghi, C.; Marchetti, M. J. Organomet. Chem. 1983, 252, 317.

(13) Itoh, K.; Nagashima, H.; Ohshima, T.; Oshima, N.; Nishiyama, H. J. Organomet. Chem. 1984, 272, 179.

(14) Young, R.; Wilkinson, G. Inorg. Synth. 1977, 17, 75.

(15) Douglas, P. G.; Shaw, B. L. J. Chem. Soc. A 1970, 1556.

(16) Coe, B. J.; Glenwright, S. J. Coord. Chem. Rev. 2000, $203,5$.

(17) Watanabe, Y.; Morisaki, Y.; Kondo, T.; Mitsudo, T. J. Org. Chem. 1996, 61, 4214.

(18) Kuwahara, T.; Fukuyama, T.; Ryu, I. Org. Lett. 2012, 14, 4703. 